\title{
BMJ Open Patients' adherence-related beliefs about methotrexate: a qualitative study of the role of written patient information
}

\author{
Charlotte Hayden, ${ }^{1}$ Rebecca Neame,${ }^{2}$ Carolyn Tarrant ${ }^{1}$
}

To cite: Hayden C, Neame R, Tarrant C. Patients' adherence-related beliefs about methotrexate: a qualitative study of the role of written patient information. BMJ Open 2015;5:e006918. doi:10.1136/bmjopen-2014006918

- Prepublication history for this paper is available online. To view these files please visit the journal online (http://dx.doi.org/10.1136/ bmjopen-2014-006918).

Received 14 October 2014 Revised 16 January 2015 Accepted 25 February 2015

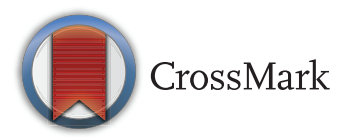

${ }^{1}$ Department of Health Sciences, University of Leicester, Leicester, UK ${ }^{2}$ Department of

Rheumatology, Leicester

Royal Infirmary, Leicester, UK

Correspondence to Dr Carolyn Tarrant; ccp3@le.ac.uk

\section{ABSTRACT}

Objectives: Methotrexate is effective in treating inflammatory arthritis, but both underadherence and overadherence can put patients at risk. Patients may fail to adhere due to practicalities including the unusual weekly dosing regimen, but medication beliefs also play a role. This study explored how both beliefs about necessity and concerns about methotrexate become established in patients with inflammatory arthritis and how patients use information in managing their beliefs and concerns.

Design: Semistructured interviews were conducted with patients taking oral methotrexate for inflammatory arthritis. Interviews were transcribed verbatim and analysed thematically.

Setting: Participants were recruited from a single Trust in the East Midlands.

Participants: Fifteen patients (4 male, 11 female) with inflammatory arthritis.

Results: Methotrexate was commonly prescribed at the time of diagnosis; at this point, experience of illness was influential for beliefs about medication necessity. Following prescription, patients absorbed information from written and verbal sources which reinforced beliefs about necessity but also raised concerns, including fear of side effects. Over time, beliefs were modified on the basis of personal experience, particularly of medication effectiveness and side effects. Some patients described tensions and dissonance in their beliefs and experiences of methotrexate, which put them at risk of nonadherence. Patients used information-seeking and information-avoidance as strategies to resolve these tensions. The available information did little to help suppress dissonance and sometimes exacerbated it.

Conclusions: Patients' experiences of coming to terms with taking methotrexate are complex, and their experiences of dissonance are particularly problematic. Experiences might be improved by supporting patients to assess necessity (particularly in the presence of side effects) and by providing information to moderate unnecessary concerns. Improving recording and sharing of monitoring results may be one way to meet these needs.

\section{INTRODUCTION}

Methotrexate is a disease-modifying antirheumatic drug (DMARD) recommended as first-

\section{Strengths and limitations of this study}

- We used qualitative methods to provide a rich insight into how necessity beliefs and concerns about methotrexate emerge in patients with rheumatic conditions.

- Drawing on the necessity-concerns framework, we were able to identify important tensions resulting in dissonance; the study shed light on how patients use information to manage tensions in their experiences and beliefs and highlighted shortcomings in the official information that patients receive about methotrexate.

- Limitations of the study are that patients were recruited from a single site and although the sample was diverse, we were not able to interview any patients over 80 years of age, or individuals who had refused or ceased methotrexate.

- Interviews were conducted at a single time point; further longitudinal research would be merited.

line treatment for rheumatoid arthritis ${ }^{1}$ and other inflammatory arthropathies, due to its superior efficacy and tolerability compared with other DMARDs. ${ }^{2}$ Guidelines recommend that it is initiated within 3 months of symptom onset for optimal clinical results. ${ }^{1}$

Tight adherence to methotrexate is essential: poor adherence may lead to suboptimal clinical outcomes. Studies have found varying levels of adherence, from significant underuse $(59 \%)^{3}$ to overuse $(107 \%){ }^{4}$ This suggests that many patients omit, delay or reduce doses of medication, while others take methotrexate more frequently than prescribed or in higher doses, risking toxicity. The unusual weekly dosing regimen and the potential for confusion between tablets of different strengths most likely increase the risk of unintentional non-adherence. ${ }^{5}$ To try to counter these risks, a national safety-focused patient information sheet was introduced in England in 2006, with recommendations from the National Patient Safety Agency (NPSA) that it should be given to all patients with arthritis starting on methotrexate. ${ }^{6}$ 
Patients may fail to adhere to medication for a range of other reasons, including symptom severity, practical constraints in their daily life and problems with recall or understanding. Alongside these issues, patients' perceptions and beliefs play an important role in adherence. ${ }^{7}$ Intentional non-adherence occurs when patients decide not to take medication as prescribed based on their beliefs, motivations and preferences. ${ }^{8}$ The necessity-concerns framework provides a model for considering these decisions as resulting from patients weighing their beliefs about medication necessity against concerns about the risks or negative consequences. ${ }^{8}$ Multiple studies have demonstrated that better adherence is associated with higher necessity beliefs and fewer concerns about treatment. ${ }^{8} 9$ Research using quantitative measures has classified patients into four categories in terms of the balance between their beliefs about necessity and concerns (see figure 1) and evidence suggests that patients who show ambivalence, scepticism and indifference to medication are at risk of non-adherence. ${ }^{10} 11$ People with rheumatoid arthritis tend to hold strong necessity beliefs about their medications and this is particularly the case for patients with more severe symptoms, but those with significant concerns are more likely to be non-adherent. ${ }^{12}$

Research into the medication beliefs of patients about DMARDs has identified tensions between patients' feelings that they have little choice but to take them and their concerns about drugs as strong and 'toxic' and the risk of side effects. These beliefs are set in the context of individuals' understanding of their disease and judgements about medication efficacy. ${ }^{13-15}$ Other work has pointed to the importance of acquisition of information from a diverse range of sources as the basis for patients' decisions about initiating DMARDs. ${ }^{16}$ Despite this qualitative work, it is not clear how individual patients' beliefs about DMARDs originate and evolve over time, nor how they manage tensions that arise.

On the basis of a qualitative study focused on the NPSA information leaflet, this paper presents findings on how beliefs about necessity and concerns about methotrexate become established in patients with inflammatory arthritis and the sources from which they arise. Furthermore, it explores how patients use information in managing these beliefs and concerns.

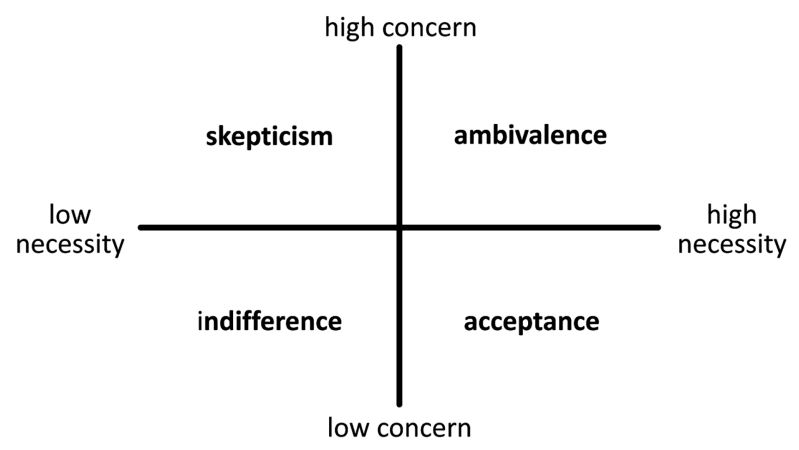

Figure 1 Variations in medication beliefs. ${ }^{11}$

\section{METHODS}

Qualitative methods, involving semistructured interviews, were used to explore the experiences of patients recently prescribed methotrexate for inflammatory arthritis-in particular, their use of information sources to make decisions about medication. Inflammatory arthropathies comprise a small number of heterogeneous conditions with varying underlying mechanisms for which methotrexate is a common treatment.

\section{Participants}

Fifteen patients were recruited from the rheumatology outpatient department of the University Hospitals of Leicester NHS Trust. Eligibility criteria for patients to be invited to interview were: over 18 years of age; diagnosed with an inflammatory arthropathy within 24 months; started on methotrexate; an appropriate level of English suitable for interview. All patients gave their written consent.

Eighty eligible patients were identified from hospital records in the outpatient setting and from a monitoring database. Efforts were made to ensure that patients who were under the care of different consultant rheumatologists were included. Patients were either approached in clinic (16) or contacted by post (64); interested patients had an opportunity to discuss the study with the researcher prior to consenting to be interviewed. Eighteen patients provisionally agreed to be interviewed, of which three did not respond to further attempts to contact. Of the remaining 62 eligible patients, 56 did not respond to the initial contact, 5 actively declined and 1 was found to be ineligible. Participants were recruited between 4 November 2013 and 27 January 2014. The sample size was limited to 15 given the time and resource constraints of this educational project, but the sample was diverse in terms of gender, age, occupation and educational background (see table 1).

\section{Data collection}

Interviews lasting approximately $1 \mathrm{~h}$ were conducted by $\mathrm{CH}$ in a quiet room on hospital premises (9 patients) or in their own home (6 patients). A topic guide was constructed based on the content of the NPSA information leaflet and incorporated questions around wider influences (see box 1). It was used to guide discussion and patients were encouraged to discuss issues that were important to them in greater depth. Interviews were digitally recorded and transcribed verbatim.

\section{Analysis}

Transcripts were analysed using the thematic approach, ${ }^{17}$ facilitated by NVivo $10 .{ }^{18}$ Familiarisation, transcript summaries and initial open coding was conducted by $\mathrm{CH}$ to identify initial themes and to inform the development of a coding tree. The first three transcripts were reviewed in detail by CT along with the remaining transcript summaries in order to offer direction to the coding tree during development. 
Table 1 Patient characteristics ( $n=$ number of participants)

\begin{tabular}{|c|c|c|c|}
\hline Age (years) & (n) & Highest qualification level* & (n) \\
\hline $20-29$ & 2 & Below 1 & 5 \\
\hline 30-39 & 0 & Levels 1-2 & 1 \\
\hline $40-49$ & 1 & Levels 3-4 & 4 \\
\hline $50-59$ & 4 & Levels 5-6 & 3 \\
\hline $60-69$ & 6 & Levels 7-8 & 2 \\
\hline $70-79$ & 2 & Total & 15 \\
\hline Total & 15 & Employment† & (n) \\
\hline Gender & (n) & Managerial, administrative and professional occupations & \\
\hline Male & 4 & Higher & 0 \\
\hline \multirow[t]{2}{*}{ Female } & 11 & Lower & 3 \\
\hline & & Intermediate occupations & 1 \\
\hline Total & 15 & Small employers and own account workers & 0 \\
\hline Ethnicity & $(n)$ & Lower supervisory and technical occupations & 2 \\
\hline Caucasian British & 13 & Semiroutine occupations & 1 \\
\hline Other & 2 & Routine occupations & 2 \\
\hline Total & 15 & Never worked, unemployed and full-time study & 1 \\
\hline Diagnosis & $(n)$ & Sick leave & 2 \\
\hline Rheumatoid arthritis & 13 & Retired & 3 \\
\hline Psoriatic arthritis & 1 & & \\
\hline Mixed/unspecified & 1 & & \\
\hline Total & 15 & Total & 15 \\
\hline Disease duration (months) & $(n)$ & Treatment duration (months) & (n) \\
\hline Up to 2 & 2 & Up to 2 & 4 \\
\hline $3-4$ & 5 & $3-4$ & 5 \\
\hline $5-6$ & 3 & $5-6$ & 3 \\
\hline $7-8$ & 1 & $7-8$ & 0 \\
\hline $9-10$ & 0 & $9-10$ & 0 \\
\hline $11-12$ & 1 & $11-12$ & 1 \\
\hline$>12$ & 3 & $>12$ & 2 \\
\hline Total & 15 & Total & 15 \\
\hline
\end{tabular}

Key overarching themes of necessity beliefs and concerns emerged strongly through the first few interviews and were noted to differ and evolve throughout the patient journey; thus, subsequent analysis was informed, but not constrained, by the necessity-concerns model. Themes relating to the use of, and need for, information were grounded in the data. As such, the analysis included both inductive and deductive elements.

A final thematic coding framework was agreed on between CT and $\mathrm{CH}$. Themes were organised into the stages of the patient journey: leading up to diagnosis, initiating treatment and maintaining treatment. Ongoing validation was ensured by personal reflections, concept mapping and ongoing discussion between $\mathrm{CH}$ and CT. Disconfirming cases were sought and used to inform the analysis. All data were coded to the final framework, data summaries were produced and data were charted using Microsoft Excel 2010 to map participants' experiences to the key concepts identified.

\section{RESULTS}

Participants described how their beliefs about the necessity of medication and their concerns emerged and evolved alongside unfolding experiences once they started on methotrexate. For some patients, tensions arose which prompted attempts at resolution: through information-seeking in attempts to assess necessity and moderate concerns, or information-avoidance to distance themselves from concerns. Both of these strategies could be problematic.

\section{The pathway to methotrexate prescription}

Prior to diagnosis, all patients described struggling with physical symptoms such as stiffness and pain. At the point of diagnosis, patients' experiences of their illness impacted on their willingness to initiate treatment and their beliefs about the necessity of medication. Many patients felt that they had reached a point at which 'something had to be done' about their condition and saw medication as a necessary next step. Pain and physical limitations were most commonly described as contributing factors towards needing and wanting to start medication.

I'd got to do something because as I say the pains in my hands, arms and everywhere, I just couldn't wait to get something to get rid of that pain.--P12

In contrast, some patients were initially reluctant to start medication due to a less severe perception of disease 


\section{Box 1 Overview of the topic guide used in interviews.}

\section{Background}

- How long have you been diagnosed with rheumatoid arthritis?

- Roughly when were you started on methotrexate treatment?

About the decision to take methotrexate

- Tell me about when you were offered methotrexate as a treatment for your arthritis.

- Can you describe to me how were given the information leaflet?

- Did you show it to family/friends?

- Tell me about the decision to start methotrexate-how was the decision made?

- How you value the leaflet in helping you making your decision?

- Did you get information from anywhere else that helped you make the decision about whether to star methotrexate?

Once you had started the treatment

-What did you expect when you started taking methotrexate?

- How has your experience of taking methotrexate been?

- Now you have been taking methotrexate for a while, how important do you think it is for you take your methotrexate treatment?

- Have you had any concerns about taking methotrexate?

- Have you looked at the leaflet again since you started taking methotrexate?

- Since starting on treatment, have you looked for information about methotrexate anywhere else?

- How often have you missed a does of methotrexate?

- What would you say were the main reason for this?

- Have you considered stopping methotrexate?

- How often do you go for blood tests to check it there are any problems with you methotrexote?

The information leaflet

Do you think there is enough information in the leaftlet?

- Is there anything else you think should be included in the leaflet?

-What would you change about the leaflet?

-What would you want to keep the same?

- Would you like this information to be available in other formats?

- Is there anything else you would like to say about the leaflet?

About you

- Can I ask how old you are?

- How would you describe your ethnic background?

- Can I ask you are currently working?

What is your highest educational qualification?

and a poor understanding of the disease-modifying potential of methotrexate, leading them to question the necessity of starting what they saw as a 'strong' drug.

It sort of contradicted that I'd got a few joint pain in my hands-because that's all it was at that point-to, well

I'm starting on a chemotherapy drug!-P7

These more reluctant patients described how the need for medication had been fostered by clinicians emphasising expectations of poor prognosis without treatment and the potential benefit of methotrexate.

\section{Starting methotrexate: information absorption and medication beliefs}

Owing to pressures on clinicians to start medication early, over half of the participants were both diagnosed and prescribed methotrexate in the same consultation. All patients reported discussion with clinicians about their diagnosis and medication and each patient recalled being given a drug information leaflet. Many described feelings of shock at the diagnosis that had contributed to difficulties absorbing information. Participants tended to rely on clinicians' decisions about the best course of action.

I listened to the important bits and the rest of it was all just a blur really. [...] when he was explaining it to me I was just like, just clouded over and I was just not really listening-P6

Patients' accounts suggested that, as they began to come to terms with their diagnosis, they began seeking and absorbing information about their disease and medication. Initial understandings of the disease and its prognosis were formed from observations of other people with arthritis; they developed perceptions of arthritis as a disabling and disfiguring disease and experienced anxiety about disease progression and for some this acted to confirm necessity.

The thought that I could end up in a wheelchair, or my perception of it was 'gnarled up', [...] [name] was on sticks and she'd only just got this rheumatoid arthritis and $[\ldots]$ she was really in a terrible state.- P1

Patients also described how information about methotrexate contributed to expectations of effectiveness.

The nurse said to me, 'Oh, this is quite a strong drug that you're on. [...] I thought, 'Good!' It's doing some good!-P13

Alongside this, some patients developed concerns about medications through this initial stage of accessing and absorbing information. Information given at the time of prescribing, particularly the NPSA leaflet, primarily related to the practicalities of taking methotrexate, the importance of monitoring and potential side effects and adverse events. While patients recognised the importance of this safety-focused information, many described how it made them worry about the risks of taking the medication.

I just had a quick look at the leaflet. [...] I saw that somehow it is very dangerous to take this medicine, like there are more side-effects than the good [effects].-P8

They described managing concerns through strategies such as hopeful thinking ${ }^{19}$ to distance themselves from 
negative perceptions and focus on the hope of a positive outcome.

I was scared, definitely scared. But you always hope that you're one of the ones who don't get the symptoms.-P11

\section{Adherence to methotrexate: medication beliefs modified by personal experience}

Initial beliefs and expectations about the medication were challenged as patients initiated treatment and began experiencing varying degrees of effectiveness and side effects.

Patients' experiences of arthritis differed, as would be expected with the variable natural history of the disease and experiences on treatment. Most patients saw improvement in their condition and this tended to be interpreted as evidence that the medication was effective. When this was coupled with few side effects, patients expressed confidence that taking methotrexate was the right thing to do.

I've noticed a big difference, and the fatigue-touch wood-the fatigue has gone. [...] I'm having less pain, and I seem to have, I've gone back almost to the same energy level.-P1

Experience of, or fear of, side effects was, however, common and most patients experienced some ambivalence about taking methotrexate. Despite this, many patients described reaching a state of acceptance of the medication, based on beliefs that its necessity outweighed any side effects or risks.

The side effects are probably outweighed by the fact you don't have to walk around like an old lady because it'll help the cause.-P15

Not all patients expressed this level of acceptance of methotrexate. For some, ambivalence and uncertainty prevailed. Some patients struggled to find a need to persist on methotrexate despite not experiencing major side effects. In particular, they questioned whether improvements in their condition could confidently be attributed to methotrexate and the necessity of taking the medication continuously.

Whether it's the steroids or the methotrexate working, I don't know. [...] How do we know it's working or not? It's just a flare up, then [the inflammation] can go down. Why should I be on the methotrexate all the time when it can probably just be used when I need it?-P3

Other participants described experiencing dissonance between perceptions of effectiveness and necessity of the medication (due to their experiences of improvements in their arthritis) and debilitating side effects (or ongoing fears about side effects and adverse consequences).

Yeah but you know it did [work]. But you know, [on the] other side, other hand I got side-effects.-P2
[I] felt assured that it work[ed]. But I was very scared at the side-effects.-P11

The role of information in managing beliefs and concerns: information-seeking and information-avoidance

Theoretical work suggests that mental discomfort caused by cognitive dissonance prompts active work to suppress it. ${ }^{20}$ Participants described using strategies of informationseeking and information-avoidance in attempts to manage their uncertainty and resolve dissonance.

Some patients explained how they actively sought information about their disease and the effects of the medication to be better able to assess the necessity of the medication and to allay their concerns, but their accounts suggested that the information available to them often fell short of meeting their needs.

Verbal information from clinicians was highly valued, but tended to be focused on disease progression rather than medication efficacy or effectiveness.

He told me even if you feel like you are OK, you need to continue with these [tablets]-P8

Written information leaflets were perceived to be of little help in reinforcing expectations of benefit or allaying concerns. Participants reported that written information leaflets lacked detail of how the drug worked and specifically the nature of the benefits that they could reasonably expect. They described how a focus on risks, side effects and adverse events did little to address concerns.

It just says that it reduces inflammation, it's not a painkiller, it reduces inflammation. How it does that? I'm not very sure about really. [...] If I stopped taking it, would it still be raging on or would it be, would it be enough, you know, to stop it?-P1

Patients wanted objective evidence that the methotrexate was working for them, in their particular case and that they were not at risk of harm. Some patients perceived monitoring as having the potential to fulfil this need, but as failing to do so in practice. They reported rarely having access to results and lacked an understanding of what particular results might mean, but suggested that they would greatly value having this information.

How do you tell whether it is working or not? Is it blood tests? I don't know.-P9

I never get to know about that-whether my liver's alright or not. [...] I have got a booklet [...] I said, 'Can I get it filled in?' to the nurse at the blood centre. She said, 'No. We don't know nothing. All we do is the blood test; it goes back to the department. [...] I'd be happier if I did get them'.-P12

With official sources of information failing to meet their needs, many patients resorted to informal sources to try to make sense of their experiences and to resolve 
their uncertainty and experiences of dissonance. Other patients with arthritis were seen as a valuable information source-particularly those who had themselves overcome difficulties while taking methotrexate. Such accounts provided patients with reassurance, but equally when patients heard about others having negative experiences, this proved to be anxiety provoking.

A few of my friends-even they are on this medicine. So I was discussing it then on the phone. They says to me you know, slowly you will settle down you know. Just take them, you slowly you will settle down.-P2

[My friend] had some really nasty side-effects with it and the one he takes is the one [the doctor] told me [...] to take.-P9

Information seeking via other informal sources, such as the internet, often did little to help allay concerns and resolve dissonance, but on occasion was reported to exacerbate it by raising further concerns.

[If you look on the internet] I know it can get you worried about the bad side-effects and if you haven't actually got it, it can make you feel like you have got it.-P4

Some patients described using avoidance strategies to reduce exposure to confusing, negative and conflicting information. For some, this involved avoiding the internet and other informal sources. Others also avoided reading official patient information to avoid worry about side effects.

I thought 'I'm not going to look on the internet' because I made a decision years ago not to do that because it just scares the living daylights out of you.- $-\mathrm{P} 7$

I think sometimes you can worry yourself reading things [...] if you read the leaflets in the tablets-any tabletyou wouldn't take them, would you? Because they've all got side effects, haven't they?-P5

\section{DISCUSSION}

\section{Main findings}

This qualitative study provides insight into how medication beliefs and concerns about methotrexate emerge and are managed in the context of decisions about taking methotrexate. The study suggests that when patients start on methotrexate, initial medication beliefs and concerns are influenced by perceptions of disease and information gained from 'official' and informal sources, but necessity beliefs and hopeful thinking tend to predominate. Once patients have personal experience of taking methotrexate, their beliefs are shaped by the way they make sense of their experiences. Ambivalence is a common experience but many patients are able to balance their beliefs about medication necessity against concerns about side effects and are accepting of medication. Others experience ongoing uncertainty about necessity, or conflicts between necessity and concern beliefs. Information-seeking and information-avoidance are strategies that patients use to try to resolve this ambivalence, but both strategies can be problematic.

\section{Strengths and weaknesses}

The need for more qualitative research to understand the psychosocial elements of rheumatic conditions has been identified; ${ }^{21}$ this study contributes by generating a rich understanding of how patients' beliefs and concerns about methotrexate emerge and suggests how patients work to manage them in the context of decisions about adhering to methotrexate. A limitation is that interviews were conducted at a single time point and participants were asked to recall their experiences and beliefs and how these had changed. Participants' accounts are likely to be constructed and reconstructed based on subsequent experiences. However, these accounts represent participants' own stories of how their beliefs and concerns evolve and as such can be seen to be meaningful. Although the sample was relatively small and participants were recruited from a single rheumatology department, it was diverse and broadly representative of the inflammatory arthritis population in terms of age, although no patients over the age of 80 were interviewed. We were not able to include any patients who had refused or chosen to cease methotrexate and these patients may have very different experiences and beliefs to patients maintained on medication.

\section{Relation to existing literature}

It is well established that the balance between necessity and concerns is influential for adherence, ${ }^{9}$ but our study makes two novel contributions to the literature. First, it suggests how patients' beliefs and concerns about methotrexate emerge and are shaped by formal and informal sources of information, particularly safety-focused information provision and their own experiences, over the course of their journey. This echoes other research that suggests that patients' belief systems are complex, evolving and influenced by information from a range of sources. ${ }^{13}{ }^{14}$ Second, it identifies dissonance ${ }^{20}$ as an important consequence of tensions between patients' necessity beliefs and concerns. While those who described ongoing ambivalence faced the most significant struggle with dissonance, our findings suggest that dissonance between necessity and concern is felt by all patients, to some degree; managing and avoiding it is a constant, active process.

Previous theoretical work has identified dissonance as a prompt to information-seeking behaviours, in an attempt to restore more consistent beliefs. ${ }^{2022} 23$ Our study shows that some patients used information-seeking as a strategy to avoid or manage dissonance. Information-seeking can help suppress dissonance as it decreases uncertainty and stress. ${ }^{24}$ In the case of methotrexate, patients sought information both to reinforce their necessity beliefs and to suppress concerns. The information they found, 
however, fell short of meeting their needs. 'Official' patient information leaflets, including those from the NPSA, were significantly moderated-written in such a way as to avoid unrealistic positive expectations and therefore did little to reinforce necessity. This has been highlighted by previous studies. ${ }^{25}$ Evidence suggests that patients with rheumatoid arthritis have a high level of need for information ${ }^{26}$ and attempts to seek information have been shown to correlate with coping better with disease. $^{27} 28$ However, our study suggests that official information could elevate concerns due to its focus on side effects, adverse events and safety. Unregulated sources such as the internet have been shown to vary in accuracy and credibility ${ }^{29}$ and were seen as containing 'frightening' information. As a result, much of the information available to patients failed to support them in efforts to reduce dissonance and, in some cases, strengthened it.

Other patients avoided information as a coping strategy. It has been hypothesised that "we can seek knowledge in order to reduce anxiety and we can also avoid knowing in order to reduce anxiety". ${ }^{30}$ Information avoidance aids one to cope with conflicting or worrying information, ${ }^{19} 24$ as patients can continue in a constant state of partial knowledge to avoid distress. ${ }^{31}$ Information avoidance enabled patients to evade negative information regarding arthritis prognosis and methotrexate, though they risked missing information important for their safety.

\section{Implications for practice and future research}

Our study suggests that patients experience unmet needs for information and support in managing uncertainty and ambivalence and resolving dissonance. This indicates that attention is required to the 'official' information that patients are given, including the NPSA information leaflet and verbal information from health professionals, to help patients understand the mechanisms of action of the medication, remain optimistic about its effectiveness and manage their concerns. Patients recognised that monitoring had the potential to provide evidence of benefit and reassurance that they were not experiencing harm, but poor documentation of results in patient-held booklets and a lack of engagement by health professionals in sharing this information with patients meant that this potential was not fully met. Other research has suggested that clinicians feel documentation of monitoring results is superfluous in meeting safety standards. ${ }^{32}$ Our findings demonstrated its potential value to patients as it is personal and trusted information. Further research is required into how to optimise the recording and sharing of monitoring results with patients.

The study also indicates that patients who use information avoidance as a strategy may need additional support and information from health professionals to ensure that this strategy does not leave them at risk.
Previous research has explored the decision-making process about medication in patients with rheumatoid arthritis, ${ }^{16}$ but our study suggests that there would be value in further longitudinal work exploring the evolution and management of medication beliefs from the time of diagnosis onwards. Mixed-method research including measures of pain levels over time would provide an important insight into how experiences of pain impact on necessity and concern beliefs.

\section{Conclusion}

Patients' experiences of coming to terms with taking methotrexate are complex and experiences of ambivalence, uncertainty and dissonance are particularly problematic. Patient experience is likely to be improved by supporting patients to assess necessity (particularly in the presence of side effects) and by providing information to moderate unnecessary concerns. Improving the recording and sharing of monitoring results may be one important way to meet this need.

Acknowledgements The authors would like to thank the patients who took part in the interviews. They would also like to thank Dr Waji Hassan and Jessy Sabu for helping with patient recruitment, and Professor Graham Martin for mentor support over the course of CH's intercalated BSc project.

Contributors All the authors contributed to the study design. $\mathrm{CH}$ interviewed participants and analysed and interpreted the data with input from CT. CH wrote the first draft of the manuscript. All the authors contributed to the writing and reviewing of the manuscript and approved the final version.

Funding This work was supported by the University of Leicester intercalated BSc fund.

Competing interests None declared.

Ethics approval Ethical approval for the project was granted by the Northampton Research Ethics Committee on 28 April 2013.

Provenance and peer review Not commissioned; externally peer reviewed.

Data sharing statement No additional data are available.

Open Access This is an Open Access article distributed in accordance with the Creative Commons Attribution Non Commercial (CC BY-NC 4.0) license, which permits others to distribute, remix, adapt, build upon this work noncommercially, and license their derivative works on different terms, provided the original work is properly cited and the use is non-commercial. See: http:// creativecommons.org/licenses/by-nc/4.0/

\section{REFERENCES}

1. National Institute for Health and Clinical Excellence. Rheumatoid arthritis: the management of rheumatoid arthritis in adults. National Institute for Health and Clinical Excellence, 2009.

2. Grove ML, Hassell AB, Hay EM, et al. Adverse reactions to diseasemodifying anti-rheumatic drugs in clinical practice. QJM 2001;94:309-19.

3. Grijalva CG, Kaltenbach L, Arbogast PG, et al. Adherence to disease-modifying antirheumatic drugs and the effects of exposure misclassification on the risk of hospital admission. Arthritis Care Res 2010;62:730-4.

4. de Klerk E, van der Heijde D, Landewe R, et al. Patient compliance in rheumatoid arthritis, polymyalgia rheumatica, and gout. $J$ Rheumatol 2003;30:44-54.

5. National Patient Safety Agency. Towards the safer use of oral methotrexate. National Patient Safety Agency, 2004.

6. National Patient Safety Agency. Oral methotrexate patient information leaflet \& dosage record booklet. National Patient Safety Agency, 2006.

7. Horne R. Compliance, adherence and concordance. In: Taylor KMG, Harding G, eds. Pharmacy practice. Taylor \& Francis, 2003:165-84. 
8. Horne R, Weinman J. Patients' beliefs about prescribed medicines and their role in adherence to treatment in chronic physical illness. $J$ Psychosom Res 1999;47:555-67.

9. Horne R, Chapman SCE, Parham R, et al. Understanding patients adherence-related beliefs about medicines prescribed for long-term conditions: a meta-analytic review of the Necessity-Concerns Framework. PLoS ONE 2013;8:e80633.

10. Horne R, Parham R, Driscoll R, et al. Patients' attitudes to medicines and adherence to maintenance treatment in inflammatory bowel disease. Inflamm Bowel Dis 2008;15:837-44.

11. Aikens JE, Nease J, Donald E, et al. Adherence to maintenance-phase antidepressant medication as a function of patient beliefs about medication. Ann Fam Med 2005;3:23-30.

12. Neame R, Hammond A. Beliefs about medications: a questionnaire survey of people with rheumatoid arthritis. Rheumatology 2005;44:762-7.

13. Kumar K, Gordon C, Barry R, et al. 'It's like taking poison to kill poison but I have to get better': a qualitative study of beliefs about medicines in rheumatoid arthritis and systemic lupus erythematosus patients of South Asian origin. Lupus 2011;20:837-44.

14. Goodacre LJ, Goodacre JA. Factors influencing the beliefs of patients with rheumatoid arthritis regarding disease-modifying medication. Rheumatology 2004;43:583-6.

15. Townsend A, Backman CL, Adam P, et al. A qualitative interview study: patient accounts of medication use in early rheumatoid arthritis from symptom onset to early postdiagnosis. BMJ Open 2013;3:e002164.

16. Salt E, Peden A. The complexity of the treatment: the decision-making process among women with rheumatoid arthritis. Qual Health Res 2011;21:214-22.

17. Braun V, Clarke V. Using thematic analysis in psychology. Qual Res Psychol 2006;3:77-101.

18. QSR International. NVivo 10. 2012. http://www.qsrinternational.com/ products_nvivo.aspx

19. Leydon GM, Boulton M, Moynihan C, et al. Faith, hope, and charity: an in-depth interview study of cancer patients' information needs and information-seeking behavior. West $J$ Med 2000;173:26-31.

20. Festinger L. A theory of cognitive dissonance. Stanford University Press, 1962.
21. Paskins Z, Hassell AB. Qualitative research in RA. Rheumatology 2012;51:3-4.

22. Bartlett FC, Bartlett FC. Remembering: a study in experimental and social psychology. Cambridge University Press, 1995.

23. Wertheimer M, Wertheimer M. Productive thinking. University of Chicago Press, 1959.

24. Brashers DE. Communication in the management of uncertainty: the case of persons living with HIV or AIDS. Commun Monogr 2000;67:63-84.

25. Pincus T, Furer V, Sokka T. Underestimation of the efficacy, effectiveness, tolerability, and safety of weekly low-dose methotrexate in information presented to physicians and patients. Clin Exp Rheumatol 2010;28:S68-79.

26. Neame R, Hammond A, Deighton C. Need for information and for involvement in decision making among patients with rheumatoid arthritis: a questionnaire survey. Arthritis Rheum 2005;53:249-55.

27. Felton BJ, Revenson TA, Hinrichsen GA. Stress and coping in the explanation of psychological adjustment among chronically ill adults. Soc Sci Med 1984;18:889-98.

28. Lambert VA, Lambert CE Jr. Coping with rheumatoid arthritis. Nurs Clin North Am 1987;22:551-8.

29. Thompson AE, Graydon SL. Patient-oriented methotrexate information sites on the internet: a review of completeness, accuracy, format, reliability, credibility, and readability. $J$ Rheumatol 2009;36:41-9.

30. Maslow $\mathrm{AH}$. The need to know and the fear of knowing. J Gen Psychol 1963;68:111-25.

31. Osterberg L, Blaschke T. Adherence to medication. N Engl J Med 2005;353:487-97.

32. Fishman D. Use of the NPSA Methotrexate Shared Care Monitoring Booklet has not improved the safety of DMARD monitoring. Rheumatology 2008;47:ii28-51.

33. Office of Qualifications and Examinations Regulations. Comparing qualification levels. 2013. http://ofqual.gov.uk/help-and-advice/ comparing-qualifications/

34. Office for National Statistics. The National Statistics Socio-economic Classification (NS-SEC rebased on the SOC2010). 2010. http:// www.ons.gov.uk/ons/guide-method/classifications/current-standardclassifications/soc2010/soc2010-volume-3-ns-sec-rebased-onsoc2010-user-manual/index.htm 